\title{
Surgical Treatment of the Funnel Chest Deformation at the Children
}

\section{Digtiar Valeryi Andreevich, Kamenska Marianna Olegovna}

Dnipropetrovsk Medical Academy Ministry of Public Health of Ukraine, Dnipro, Ukraine

\section{Email address:}

mkaminska@mail.ru (K. M. Olegovna)

\section{To cite this article:}

Digtiar Valeryi Andreevich, Kamenska Marianna Olegovna. Surgical Treatment of the Funnel Chest Deformation at the Children. American Journal of Pediatrics. Vol. 3, No. 2, 2017, pp. 8-12. doi: 10.11648/j.ajp.20170302.12

Received: February 24, 2017; Accepted: March 16, 2017; Published: April 25, 2017

\begin{abstract}
Introduction: Funnel chest deformation (FCHD) is the most common malformation of the chest. This anomaly appears as the cosmetic and functional disorders of the cardiovascular and respiratory systems. Its main characteristic feature is tendency to progression, closely linked with the growth and age of children. Purpose: To analyze results of surgical treatment at the children with FCHD, using minimally invasive method of thoracoplasty. Materials and methods: The research group included 21 children with FCHD at the age from 4 to 17 years. Symmetrical shape of deformation was revealed in 14 patients (66.6\%), the asymmetric shape was revealed in $5(23.8 \%)$ patients, the FCHD - in 2 children $(9.5 \%)$. Preoperative examination included: routine clinical laboratory research, spirography, electrocardiography, echocardiography, chest radiography in the frontal and lateral projections and computed tomography. All patients underwent thoracoplasty with using method D. Nuss. Results: The period of supervision covered from 6 months to 4 years after surgery operation. Good results were obtained in 17 $(80.96 \%)$ cases; satisfactory - in 3 cases $(14.28 \%)$, unsatisfactory - in 1 case $(4.76 \%)$. Conclusion: Surgical treatment of FCHD with using innovative in Ukraine method of retrosternal metal fixators - is vital importance direction in thoracoplasty, having numerous advantages, compared with resection techniques, as well as minimally invasive and a good cosmetic effect.
\end{abstract}

Keywords: Funnel Chest Deformation, Thoracoplasty, Children

\section{Introduction}

FCHD is the most typical malformation of chest, which covers $91 \%$ of all congenital chest deformations, according to the review of different authors $[1,5]$. Characteristic feature of FCHD is tendency to progression, which is closely linked with a growth and age of children. Further development of FCHD leads to the more severe disorders of lungs, to the displacement and rotation of a heart, as a result - to the decompensation of cardiovascular and respiratory systems [4, 6]. Etiology of FCHD in the modern time is not research enough, but the major role in its development plays such factors as: hereditary predisposition, genetic defects. It should be noted, that FCHD is one of numerous phenotypic characteristics of the undifferentiated connective tissue dysplasia syndrome (UCTDS), which presence at the children has negative impact in a case of disease. Presence at the children UCTDS signs determines severity of deformation and could be used for selection the optimal terms of surgical intervention [18].
It is well - known surgical treatment of FCHD, because conservative treatment is not effective. It was offered more than 80 different variants of surgical procedures and their modifications for FCHD correction, but there is a high percentage of unsatisfactory results - to $41.7 \%$ [2, 3], which indicated about a relevant and unresolved problem in general, and to the necessity of further research in this field.

According to Petersen C. [7], variety of open surgery techniques represented the "golden standard" for remodeling the chest wall of patients with pectus excavatum. This situation changed when a minimally invasive approach was first introduced by D. Nuss. This procedure was originally developed only for children and is based on the fact that the growing chest wall is still flexible. The new technique is gaining acceptance worldwide in the field of pediatric surgery, and older patients who resist open surgery for correction of their pectus excavatum now request the new and less invasive procedure.

In the work of Barauskas V. [8] carried out a retrospective and prospective analysis performed of the methods of 
investigation and treatment of 504 patients operated for the funnel chest during the period of 35 years (1968-2002). On basis of physical examination and anamnestic data the most clinical signs and complaints were established. The heart compression was found in preoperative X-rays in 476 $(94.4 \%)$ patients, the heart was rotated and dislocated in 441 $(87.5 \%)$ patients. The following indications for the funnel chest surgical treatment were established: - obvious funnel chest diagnosed by physical examination, - complaints and disturbances clear from anamnestic data, - heart rotation and compression established by radiological examination, pathological findings in cardiorespiratory system (ECG, echocardiography, and spirography).

Kelly RE Jr. [9] study pectus excavatum indications for surgical treatment, which include two or more of the following: a severe, symptomatic deformity; progression of deformity; paradoxical respiratory chest wall motion; computer tomography scan with a pectus index greater than 3.25; cardiac compression/displacement and/or pulmonary compression; pulmonary function studies showing restrictive disease; mitral valve prolapse, bundle branch block, or other cardiac pathology secondary to compression of the heart; or failed previous repairs.

In the work of other authors [10], discuss the results of pulmonary function tests performed at rest in 25 patients treated for pectus excavatum. Surgical correction of the lesion was performed by resection of the deformed costal cartilages, longitudinal and transversal sternotomy, stabilization of the anterior chest wall with Kirschner wires. Control tests carried out 6 months following the surgical correction. In cases of severe deformation of the chest wall a decrease of pulmonary function indices was evident.

Other works [11], were devoted to the research of pectus excavatum in adult patients, often causing psychological complaints and physiological impairments. Although lung function at rest may minimally deteriorate after surgical correction, it remains unclear if surgery improves exercise capacity. A prospective study was performed to compare pulmonary and cardiovascular function at rest and at maximal exercise, before, and at 1 year after pectus excavatum repair.

Group of authors [12] study a funnel chest and pigeon chest, which are nosologically a uniform entity. Psychocosmetic reasons are recognised as indication for operation. The optimal age for operation is the second to the sixth year of life. During 20 years, 765 patients were operated on at our hospital without lethality. Postoperative complications were pneumothorax $(4 \%)$, pneumonia $(2 \%)$, after bleeding (2\%) and disturbed wound healing (7\%). Late results 5 years following surgery were excellent in $57 \%$, good in $27 \%$, satisfactory in $10 \%$ and unsatisfactory in $6 \%$ of the cases, thus adding up to $84 \%$ good results.

Nakanishi Y. et al. [13], had refined the Ravitch technique of sternal elevation for surgical correction of funnel chest. The authors' major modification is introducing a living rib strut for supporting the elevated sternum. The left 5th rib with a vascular pedicle from the internal thoracic artery is turned 180 degrees beneath the sternum. We have used this method in 10 cases. The results have all been satisfactory.

Ishikawa S. et al. [14] have improved the sternal turnover surgical procedure by using a vascular pedicle for a funnel chest. The following method for performing the operation was devised: (1) cutting the deformed sternum at the second intercostal position; (2) cutting the second costal cartilages to allow a repositioning of the vascular pedicle onto the presternal surface; (3) turning the sternum over, placing one end on the other, and attaching one end to the other; and (4) making a groove in the turned-over sternum to prevent the decussated vascular pedicle from becoming constricted. By using this method, it is easily possible to keep the bilateral internal thoracic vessels intact.

According to Zhang XG. [15], the experience of using a modified sternal turnover technique in 4 patients with pectus excavatum in which one or both internal mammary vascular pedicles were preserved to assure viability of the sternum and costal cartilages in the everted position. The internal mammary vessels were carefully dissected upward and downward each for $5 \mathrm{~cm}$ and the funnel shaped sternum was turned over for 180 degrees with care being exercised not to injure the blood vessels. Author recommended this technique the first choice for the repair of pectus excavatum.

Kilda A. et al. [16] study the sternovertebral distance, which was significantly increased after Nuss operation. Anteroposterior and lateral chest radiographs were performed before Nuss procedure, 1, 6, and 12 months after operation and finally 1 month after bar removal. A total of 84 children with funnel chest were operated on. Preoperative sternovertebral distance was $79.81+/-6.96 \mathrm{~mm}$; 1 month after operation, $97.84+/-17.08 \mathrm{~mm}$; 6 months, $110.55+/-13.85 \mathrm{~mm}$; and 12 months, $113.6+/-14.61 \mathrm{~mm}$.

$\mathrm{Wu} \mathrm{TH}$. et al. [17] study was devoted to evaluation of chest radiographs, which offer useful information for the assessment of pectus excavatum before and after Nuss repair in adults. The preoperative mean Haller indices measured on computed tomography and chest radiographs were $4.61 \pm$ 1.58 (range, 2.6-11.9) and $3.82 \pm 1.17$ (range, 2.0-10.2), respectively. The postoperative mean Haller index measured on chest radiographs was $2.86 \pm 0.56$ (range, 1.7-5.4). The mean sternovertebral distances detected on preoperative and postoperative ( $>6$ months after surgery) lateral chest radiographs were $7.67 \pm 1.89 \mathrm{~cm}$ (range, $2.5-12.9 \mathrm{~cm}$ ) and $9.89 \pm 1.80 \mathrm{~cm}$ (range, 4.6-15.0 cm). The mean sternovertebral distance in patients after the bar removal detected on lateral chest radiographs was $9.25 \pm 2.14 \mathrm{~cm}$.

Our research aimed to analyze results of the surgical treatment of FCHD at the children with using minimally invasive method of thoracoplasty.

\section{Materials and Methods}

Results of our observation and treatment, which carried out in 21 children with FCHD, aged from 4 to 17 years have been shown, that there were examined $17(80.95 \%)$ boys and $4(19.05 \%)$ girls (table 1). 
Table 1. Subdivision of patients by age and gender.

\begin{tabular}{lllll}
\hline \multirow{2}{*}{ Gender } & Age & & \multirow{2}{*}{ Totally } \\
\cline { 2 - 4 } & 4-9 years & $\mathbf{1 0 - 1 4}$ years & $\mathbf{1 5 - 1 8}$ years & \\
\hline Boys & 8 & 5 & 4 & 17 \\
Girls & 1 & 3 & - & 4 \\
Totally & 9 & 8 & 4 & 21 \\
\hline
\end{tabular}

In order to confirm UCTDS in all patients we used international phenotypic scale by M. G. Glesby (1989) (table 2).

Table 2. Structure of the phene signs, according to the phenotypic map of $M$. G.Glesby (1989).

\begin{tabular}{|c|c|c|c|}
\hline № & Signs & № & Signs \\
\hline 1 & Asthenic type of constitution & 9 & Gothic palate \\
\hline 2 & Loss of normal posture & 10 & $\begin{array}{l}\text { Hypermobile joint } \\
\text { syndrome }\end{array}$ \\
\hline 3 & Scoliosis of the spine & 11 & Increased skin extensibility \\
\hline 4 & Straight back & 12 & $\begin{array}{l}\text { Multiple pigmented spots } \\
\text { on the skin }\end{array}$ \\
\hline 5 & $\begin{array}{l}\text { Keel-shaped deformation of } \\
\text { the chest }\end{array}$ & 13 & $\begin{array}{l}\text { Positive symptom of } \\
\text { "wrist" }\end{array}$ \\
\hline 6 & $\begin{array}{l}\text { Funnel-shaped chest } \\
\text { deformation }\end{array}$ & 14 & $\begin{array}{l}\text { Positive symptom of } \\
\text { "thumb" }\end{array}$ \\
\hline 7 & Arachnodactylia & 15 & Myopia \\
\hline 8 & Flat feet & 16 & Ectopia of the lens \\
\hline
\end{tabular}

In all examined children have been revealed: polysystemic lesions of connective tissue at least in two systems, at 2/3 of the given patients polysystemic lesions were observed in three systems, $19.04 \%$ of patients having polysystemic lesions in four systems of the human body. External phenotypic UCTDS signs should be divided into three basic groups: skeletal, articular abnormalities, and cutaneous manifestations. The data analysis shows, that children with early severe forms of congenital deformities of the chest among UCTDS phenotypic signs dominated the skeletal changes, which were detected in 22 cases $(68.75 \%)$. The articular abnormalities were revealed in 13 patients $(40.6 \%)$, the skin manifestations in 6 patients $(18.75 \%)$.

The greatest rate of progression was in puberty. In this period, at the children was forming a gross cosmetic defect, accompanied by disorders of cardio-pulmonary activity, which had been shown a direct indication to the operative treatment. Majority of children were operated at the age from 9 to 15 years, when deformation of chest had the most manifestoes clinical symptoms. In all patients FCHD was diagnosed from an early age. According to our observations, FCHD did not always have a symmetrical configuration. It was revealed $14(66.6 \%)$ patients with symmetrical form of deformation, $5(23.8 \%)$ patients with asymmetrical form of deformation, 2 (9.5\%) patients - with FCHD. Different by the structure and severity spinal deformations in the sagittal and frontal plane was observed in $16(76.2 \%)$ children. Preoperative examination included conduction of the clinical laboratory tests, spirography, electrocardiography, echocardiography, X-ray chest examination in the frontal and lateral projections, computed tomography - by individual indicators. Conducted examination helped to identify ventilation and respiratory disfunctions in the lungs. According to the data of spirography, a lung capacity was decreased, and function of external respiration by restrictive or obstructive type was impaired among $71.43 \%$ of children. At the oldest age group of children was dominated restrictive type of the respiratory failure, primary, because of limiting the mobility of ribs, diaphragm and reducing volume of air. Significant changes were observed in the cardiovascular system on the ECG examination. It was revealed rhythm disturbance in a form of bradycardia and tachycardia at the 28 and $57 \%$ of children. Phenomenon of conduction disorders having $71.43 \%$ of patients in the form of atrioventricular and incomplete blockade in a right bundle of His, overload in the right side of heart. Echocardiography allows to specify morphological changes in the heart, in $52.38 \%$ of cases were observed a mitral valve prolapse. Radiologically was determined degree of deformation of the thorax, using index of Hizhitska. It was diagnosed II stage $8(38.1 \%)$ and III stage - 13 (61.9\%). A computed tomography was performed in order to determine relative position of mediastinum to the sternocostal complex, and for purpose of assessment the brunch - pulmonary system we calculated Haller index.

The main indications for performing of thoracoplasty:

1. Hizhitska index should be less than 0.6 or CT index of Haller more than 3.2.

2. Visible cardiac abnormalities in the EKG and ultrasound investigation; reduction of a lung capacity, according to the data of spirography, presence of atelectasis.

3 . The documented rapid progression of the deformation.

In all cases was conducted a surgical treatment. In 16 patients we used thoracoplasty, according to the method of D. Nuss. In 5 cases we additionally performed osteotomy of a sternum and chondrotomy of the ribs.

Operation was performed by the following way: position of the patient in the back. Hands were situated in the shoulder joints up and to the side at the angle 110 degrees. We performed a transverse skin incision from the front to the back axial line in the level of maximum deformation of a chest. It was separated a skin flap to the parasternal line symmetrically on both sides. To the incision in the left half of a chest was introduced a special tool - conductor in the direction from the left to the right and carried out it through retrosternally space to the exit from the opposite side of a slit. Hereafter, it was conducted a lavsan thread. To the lavsan thread was fixed a plate, which was constructed, taking into account optimal correction of the thoracic deformations. At the traction for a thread - in a direction from the left to the right, we formed the tunnel, which carried out a plate bending backwards to the release of it from the slit in the right. The powerful clips captured a plate for the distal departments from both sides, after that performed a rotation to $180^{\circ}$. Plate was placed on the anterior ribs. It was fixing to the muscular part of subordinate ribs in 4 points from both sides. We checked the stability of a striker. Wounds were sutured in layers. During the operation, control of the retrosternal conduction a metal latch was carried out through minimal incision in the region of xiphoid outgrowth or with using thoracoscopy. In all cases were carried out the 
combined endotracheal anesthesia. In 30 minutes before incision, there was intravenously administered daily dose of antibiotics (vancomycin). Infection of a base plate was not revealed.

\section{Results and Discussion}

Results of thoracoplasty by application method of D. Nuss were assessed in 21 patients. For the assessment of clinical efficiency of surgical treatment patients with FCHD we analyzed results in the early postoperative and remote periods (after removal of the metal constructions).

Results of surgical treatment in the early postoperative period were evaluated according to the intraoperative blood loss, duration of surgery, duration of anesthesia, narcotic analgesics, term of verticalization of the patients, presence of complications.

At the patients, operated by this technique, were not performed hemo- transfusions of the red blood cell and blood products. Intraoperative blood loss in the operated patients was varied from 50 to $80 \mathrm{ml}$ during the skin incision and forming a space for conduction a plate and its fixation. Average duration of surgery was ranged from 60 to 110 minutes. Anesthesia was conducted with narcotic analgesics for 1-2 days and then with non-narcotic analgesics for 5-7 days. Using of epidural anaesthesia improved at the 5 patients the analgesic effect. Catheter was removed on the $2-$ 3 day. Majority of children were transferred to a vertical position, which allowed them to start walking on the 2 nd day.

Among the complications at the early postoperative period were hemothorax and pneumothorax. Hemothorax was observed in $7(23.8 \%)$ patients. In the ultrasonography was revealed on average $100 \mathrm{ml}$ of fluid. In $2(9.52 \%)$ cases were performed thoracocentesis as the passive drainage of pleural cavity, in 4 (19.04\%) children was carrying out a pleural puncture. In one patient was developed an exudative plevritis and pericarditis, which were blocked by applying drainage of the pleural cavity and pericardium, a long-term antibiotic therapy.

In the long-term postoperative period at the 3 patients $(14.28 \%)$ was appeared seromes in the region of postoperative wound on the right. Their elimination was carried out by excision of the pathologically changed tissues, imposition of the secondary sutures, antibiotic therapy.

To the complications in a long-term period was carried out instability of the implant. In $2(9.52 \%)$ patients was observed shift of the metal construction, caused by its deformation in 1 month and after 1.5 months after surgery. The urgent patients was performed the surgery - it was removed a plate. In one case was conducted the surgery after 8 months without any complications. In the second case, from repeated operations declined, resulting in a relapse of the deformity. In the second case, a patient was not agreeing to the surgery, as the result we found out relapse of the given deformations. This case was related to the unsatisfactory results.

Long-term results of treatment have been traced in a period from 6 months to 4 years after surgery. We evaluated results of the surgery on the following criteria: good (deformation is eliminated completely, anthropometric indices correspond to the age, cosmetic result is satisfied the patient, functional studies do not reveal deviations from the norm); satisfactory (a partial recurrence of the deformation, the index of Hizhitska should not be less than 0.7, or curvature of the ribs, complaints on cosmetic defects are missing, or minimal, functional parameters are normal); unsatisfactory (complete relapse of the deformations, complaints on the cosmetic defect). At the research group of patients good results were obtained in 17 (80.96\%) cases, satisfactory - in $3(14.28 \%)$ cases, unsatisfactory - in 1 $(4.76 \%)$ case.

Therefore, innovative thoracoplasty technique, which we conducted by the method of $\mathrm{D}$. Nuss with using retrosternal retainer is the less traumatic, than traditional methods of treatment. It allows us to reduce duration of postoperative staying of patient in the hospital and obtain good cosmetic results. Analysis of postoperative complications and unsatisfactory results of treatment showed, that the given method required improvement of a quality of applied metal clamps in order to increase their strength properties, as well as technical support the possibility of their modeling during the operation.

We proposed our first experience of application method of D. Nass, while in the literature review have been shown a numerous cases of operated patients. However, we consider that any case of FCHD should be corrected with using this method. Unsatisfactory result, at the moment of the plate removal, was observed in a case of children with visible phenotypic signs of UCTDS.

\section{Conclusion}

We consider age of children 8-12 years should be optimal for surgery, because the chest is elastic and easier could be corrected. Primary, we achieved a proper formation of the chest. Finally, we prevented development of the functional disturbances and secondary deformations of the chest and spine. In a case of rapidly progressive congenital deformities of the chest with severe forms of UCTDS, an early surgical intervention (in the age 4-6 years) is the basis for normal development of cardio - pulmonary system. Surgical correction of the funnel chest deformation with using metal clips retrosternally should be the innovative direction of thoracoplasty, having a numerous advantages in comparison with resecting techniques. Among them should be the minimally invasive way and good cosmetic effect.

\section{References}

[1] Huba AD. Surgical treatment of the funnel chest deformation in the children and adolescents: Abstract of dissertation for PhD degree, Kurgan, 2007; 18.

[2] Krivchenia D.Yu. et al. Variants of modifications the Nuss operation in a correction of the funnel chest deformation: advantages and disadvantages, Surgery of children's age, 2011, 1: 27-30. 
[3] Malakhov OA, Zherdev KV, Chelpachenko OB. Orthopedic aspects and features of surgical treatment of the funnel chest deformation in the children and adolescents, Bulletin of traumatology and orthopedics, named by N. N. Priorova, 2011, 3: 3-9.

[4] Plyakin VA, Kulik IO, Sarukhanyan OO. Comparative assessment of the operations by Nass and Ravich for treatment of the funnel chest deformation, Pediatric surgery, 2013, 3: 6064 .

[5] D. Nuss, R. E. Kelly Jr., D. P. Croitoru, M. E. Katz. A 10-year review of a minimally invasive technique for the correction of pectus excavatum, J. Pediatr. Surg., 1998, Vol. 33 (4): 545552 .

[6] S. Hosie, T. Sitkiewecz, C. Petersen et al. Minimally invasive repair of pectus excavatumthe Nuss procedure. A European multicentre experience, Eur. J. Pediatr. Surg., 2002, Vol. 12 (4): $235-238$

[7] Petersen C. Funnel chest. New aspects since introduction of a minimally invasive surgical technique, Orthopade. 2003 Oct; 32(10):916-9.

[8] Barauskas V. Indications for the surgical treatment of the funnel chest, Medicina (Kaunas). 2003; 39(6):555-61.

[9] Kelly RE Jr. Pectus excavatum: historical background, clinical picture, preoperative evaluation and criteria for operation, Semin Pediatr Surg. 2008 Aug; 17(3):181-93.

[10] Kowalewski J, Barcikowski S, Zołyński K. Effect of correcting funnel chest on respiratory function, Pneumonol Alergol Pol. 1993; 61(1-2):35-9.
[11] Neviere R, Montaigne D, Benhamed L, Catto M, Edme JL, Matran R, Wurtz A. Cardiopulmonary response following surgical repair of pectus excavatum in adult patients. Eur J Cardiothorac Surg. 2011 Aug; 40(2): 77-82.

[12] Hecker WC, Happ M, Soder C, Remberger K, Nehrlich A. Clinical aspects and problems of pigeon breast and funnel chest, Z Kinderchir. 1988 Feb; 43(1):15-22.

[13] Nakanishi Y, Nakajima T, Sakakibara A, Nishiyama T. A vascularised rib strut technique for funnel chest correction, $\mathrm{Br}$ J Plast Surg. 1992 Jul; 45(5):364-6.

[14] Ishikawa S, Uchinuma E, Itoh M, Shioya N. A simple sternal turnover procedure using a vascular pedicle for a funnel chest, Ann Plast Surg. 1988 May; 20(5):485-91.

[15] Zhang XG. Sternal turnover with preservation of internal mammary vessels in the treatment of pectus excavatum, Zhonghua Wai Ke Za Zhi. 1989 May; 27(5):282-3, 317.

[16] Kilda A, Lukosevicius S, Barauskas V, Jankauskaite Z, Basevicius A. Radiological changes after Nuss operation for pectus excavatum, Medicina (Kaunas). 2009; 45(9):699-705.

[17] Wu TH, Huang TW, Hsu HH, Lee SC, Tzao C, Chang H, Cheng YL. Usefulness of chest images for the assessment of pectus excavatum before and after a Nuss repair in adults, Eur J Cardiothorac Surg. 2013 Feb;43(2):283-7.

[18] Smits-Engelsman B., Klerks M., Kirby A. Beighton score: a valid measure for generalized hypermobility in children. J. of Pediatrics. 2011; 158 (1): 119-123. 